\title{
Prediction of Stress-Strain Behaviors in Steels Using an Integrated Constitutive, FEM and ANN Model
}

\author{
L. X. KONG, B. WANG ${ }^{11}$ and P. D. HODGSON \\ School of Engineering and Technology, Deakin University, Geelong Vic 3217 Australia. \\ 1) Department of Mechanical Engineering, Brunel University, Uxbridge, London UB8 3PH UK.
}

(Received on December 13, 2000; accepted in final form on March 7, 2001)

\begin{abstract}
Austenitic steels with a carbon content of 0.0037 to 0.79 wt $\% \mathrm{C}$ are torsion tested and modeled using a physically based constitutive model and an Integrated Phenomenological and Artificial neural Network (IPANN) model. The prediction of both the constitutive and IPANN models on steel $0.017 \mathrm{wt} \% \mathrm{C}$ is then evaluated using a finite element (FEM) code ABAQUS with different reduction in the thickness after rolling through one roll stand. It is found that during the rolling process, the prediction accuracy of the reaction force from FEM simulation for both constitutive and IPANN models depends on the strain achieved (average reduction in thickness). By integrating FEM into IPANN model and introducing the product of strain and stress as an input of the ANN model, the accuracy of this integrated FEM and IPANN model is higher than either the constitutive or IPANN model.
\end{abstract}

KEY WORDS: finite element methods; constitutive model; artificial neural networks; hot strength; simulation.

\section{Introduction}

Development of a model to accurately predict the strainstress behavior is very important to set up the millgap for rolling process. Over the years different models have been developed including constitutive, ${ }^{1)}$ empirical ${ }^{2)}$ and $\mathrm{ANN}^{3)}$ models. For example, Collinson et al. ${ }^{4)}$ studied the effect of carbon content on the flow strength, static and dynamic recrystallisation behavior of carbon steels with carbon content ranging from 0.0037 to $0.79 \%$. It was found that the activation energy $Q_{\text {def }}$ varied with the carbon content and consequently, no simple trend could be developed for all of the steels studied. Based on this, an Integrated Phenomenological Artificial Neural Network (IPANN) model has been developed ${ }^{3,5)}$ with the effect of both the deformation conditions and material composition on the strain-stress behavior being more accurately predicted. IPANN significantly improves the prediction accuracy of hot strength in both work hardening and dynamic recrystallisation regimes. ${ }^{5}$

Using a 2-D Finite Element (FEM) model, a plate with a thickness of $32 \mathrm{~mm}$ being rolled through a single roll stand was modeled by Kong et al. ${ }^{6}$ The material properties of an austenitic steel $0.017 \mathrm{wt} \% \mathrm{C}$ measured with hot torsion machine and predicted with Estrin and Mecking (EM) constitutive model and IPANN model were used. Although the standard error of the IPANN model is lower than the EM model, it is found that the prediction of the reaction force and torque from FEM simulation is less accurate for IPANN model. It is the summary of the product of strain and stress in the deformation range, which contributes most significantly to the precise prediction. ${ }^{6)}$ However, only one simulation was conducted in $^{6}$ and whether this observation can be applied to a wider range of deformation needs to be further investigated.

In the present work, the strain-stress relation measured from torsion test and predicted with constitutive model and IPANN model is used as material property in the FEM code ABAQUS to simulate a rolling process with deformation corresponding to three cases ie the area of the experimental strain stress curve closer to IPANN, nearly in the middle of generalized EM model and IPANN, and closer to generalized EM model. An integrated FEM and IPANN model is then developed and used to predict the strain-stress behavior of these steels.

\section{Materials}

Steel with a carbon content of 0.0037 to $0.79 \mathrm{wt} \%$ was studied. $^{4,5}$ The steels were cast in a vacuum furnace as 65 $\mathrm{kg}$ ingots before being hot rolled, machined into torsion samples and torsion tested using the hot torsion machine. The austenite grain size prior to testing was close to 100 $\mu \mathrm{m}$ for all steels and tests were performed at temperatures of 900,1000 and $1100^{\circ} \mathrm{C}$ and at strain rates from 1 to 100 $\mathrm{s}^{-1}$.

The strain-stress behavior is typical of steels deformed in the austenite phase region showing work hardening up to a peak stress followed by softening, indicative of dynamic recrystallisation. Although it is found that that stress increases with an increase in strain rate and a decrease in temperature for all steels as expected, the carbon content 
presents a very complex effect on stress-strain curves. ${ }^{4)}$

\section{Model Development}

\subsection{Constitutive Model}

The strain-stress behavior of steels can be predicted with many models. Using a model developed by Estrin and Mecking $(\mathrm{EM})^{7)}$ for working hardening area and integrated Avrami equation $^{8)}$ into EM model for recrystallisation regime, the stress can be expressed as,

$$
\begin{array}{ll}
\sigma^{\mathrm{EM}}=\left[\sigma_{\mathrm{s}}^{2}+\left(\sigma_{0}^{2}-\sigma_{\mathrm{s}}^{2}\right) \exp (-2 B \varepsilon)\right]^{1 / 2} & \varepsilon<\varepsilon_{\mathrm{c}} \\
\sigma=\sigma^{\mathrm{EM}}-X\left(\sigma^{\mathrm{EM}}-\sigma_{\mathrm{ss}}\right) & \varepsilon>\varepsilon_{\mathrm{c}}
\end{array}
$$

where $\sigma_{0}$ : initial stress when $\varepsilon=0$;

$B$ : a constant which is a function of ZenerHollomon parameter;

$\sigma_{\text {ss: }}:$ the steady state stress;

$X:$ the fraction recrystallised at a specific strain; and

$\varepsilon_{\mathrm{c}}$ : critical strain which is often replaced with peak strain, $\varepsilon_{\mathrm{p}}$, due to difficulty in accurately determining this in some tests.

\subsection{Integrated Phenomenological Artificial Neural Networks (IPANN)}

By incorporating the work hardening coefficient $(\theta)$ and the square root product of $\theta$ and $\sigma(\sqrt{\theta \sigma})$ with the ANN model, Kong and Hodgson ${ }^{9)}$ developed an Integrated Phenomenological Artificial Neural Network (IPANN) model. The IPANN model improved the prediction accuracy of carbon steels. ${ }^{5)}$ As the activation energy $Q_{\text {def }}$ was different for steels studied in this work, the Zener-Hollomon parameter was a parameter to represent the effect of activation energy and deformation. In IPANN model, three more parameters, $\log (Z)$, work hardening rate $\theta$, and $\sqrt{\theta \sigma}$ were used as inputs in the ANN model to predict the stress in addition to the deformation conditions, i.e. strain, strain rate and temperature, and material composition ie carbon content. It should be noted that the experimental data must be analyzed to obtain $Z, \theta$, and $\sqrt{\theta \sigma}$ for the training and test data from the physical models.

The prediction of both the constitutive and IPANN models is compared with the torsion test of a steel with carbon content of $0.017 \mathrm{wt} \%$ deformed at a temperature of $900^{\circ} \mathrm{C}$ and a strain rate of $1 \mathrm{~s}^{-1}$ in Fig. 1. For IPANN model, the prediction is more accurate than the generalized EM model in both the work hardening and dynamic recrystallisation regimes. However, the IPANN underestimates the stress in both the low and high strain regions. Although the average of absolute deviations for both the EM (73.4) and IPANN (73.5) models is almost the same, the standard error for the prediction of IPANN model (2.1) is much lower than that of the EM model (4.9). Therefore, the IPANN model is more accurate than EM constitutive model from the statistical information of both the constitutive and IPANN models.

\subsection{Finite Element Model}

The hot rolling process was modeled using the commercial FEM code ABAQUS to examine the influence of models' accuracy. A 2-dimensional analysis was carried out in this study on a steel plate with a thickness of $40 \mathrm{~mm}$ and a length of $180 \mathrm{~mm}$. The radius of the roll, which rotates at a

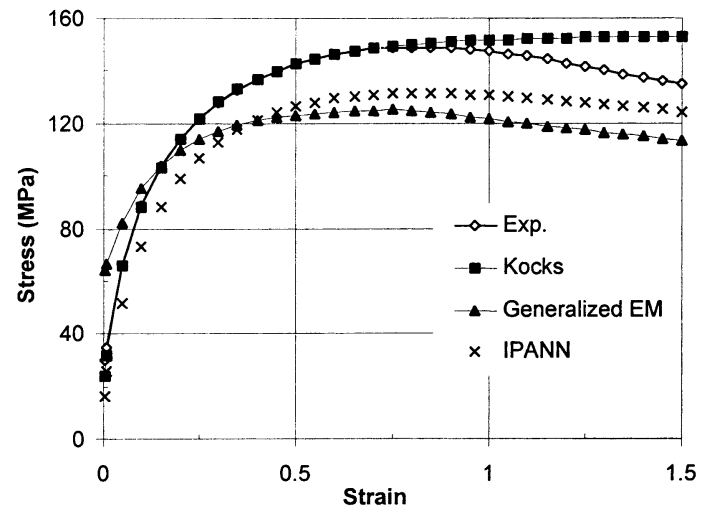

Fig. 1. Comparison of the prediction of constitutive and ANN models with experiment. (Carbon content: $0.017 \mathrm{wt} \%$, temperature: $900^{\circ} \mathrm{C}$, strain rate: $1 \mathrm{~s}^{-1}$ )



Fig. 2. Variation of area under strain-stress curve with strain. (Carbon content: $0.017 \mathrm{wt} \%$, temperature: $900^{\circ} \mathrm{C}$, strain rate: $1 \mathrm{~s}^{-1}$ )

speed of $60 \mathrm{rpm}$, is $170 \mathrm{~mm}$ and modeled as a rigid body. The experimental results and EM constitutive and ANN predictions (Fig. 1) for the steel of $0.017 \mathrm{wt} \% \mathrm{C}$ deformed at a temperature of $900^{\circ} \mathrm{C}$ and a strain rate of $1 \mathrm{~s}^{-1}$ were used as material strain-stress data. A Poisson's ratio of 0.3 was assumed. No rate dependence and temperature dependence were taken into account.

Coulomb friction was originally assumed between the roll surface and the plate, with a friction coefficient of 0.3 . Due to the symmetric nature, only a half of the plate was modeled with 819 nodes and 720 elements.

The plate was simulated by being rolled through one roll stand and was reduced to its 90,80 and $70 \%$ thickness. The real strain in the plate after deformation varies with the position and in some areas is much bigger or smaller than the average strain. ${ }^{6}$ As indicated by Kong et al., the reaction force is an indicative of the energy consumed during the rolling process and can be related to the deformation energy required and, ${ }^{\text {) }}$

$$
W \propto \sum_{\varepsilon=0}^{\varepsilon_{\mathrm{Ave}}} \sigma \times d \varepsilon
$$

where $W$ : the energy needed to deform a unit volume of material;

$\varepsilon_{\mathrm{A}_{\mathrm{ve}}}$ : the average deformation needed to be achieved.

The variation in the product of strain and stress with the 
deformation range is shown in Fig. 2.

\subsection{Integrated FEM and IPANN Model}

From the FEM prediction, it was found that the area under strain stress curve is another important parameter representing the material deformation properties in addition to the work hardening coefficient and Zener-Hollomon parameter. ${ }^{6)}$ In this work, the area is integrated into the IPANN model as an additional input.

The training strategies and data preparation followed those discussed in previous work. ${ }^{3,9)}$ The test data not used in the training is then employed to verify the accuracy of the integrated model.

\section{Results and Discussion}

\subsection{Simulation of Finite Element Model}

Although the prediction error for both the EM and IPANN models is not significant in comparison with the experimental data, the history of the reaction force on the rolls from the roll starting point to the steady state and the steady reaction force exhibit an obvious difference for three different material properties used in the simulation. ${ }^{6}$ ) Generally, the reaction force increases gradually when the plate feeds into the millgap as the deformation at this stage is very small. However, after the rolls rotate about $45^{\circ}$, the reaction force increases rapidly to a steady value. For the strain-stress behavior from experiment, EM and IPANN models, the time to achieve the steady state is almost the same. ${ }^{6}$ )

From the distribution of predicted equivalent plastic strain with a reduction of $10 \%$ in thickness (Fig. 3), it is found that the pattern for all three strain stress curves is similar. However, the higher strain area of IPANN is bigger than that of the experiment and generalized EM model because IPANN model underestimated the stress.

Consequently, the reaction force is smaller for the prediction using IPANN strain stress curve with an average reduction in reaction force of $19 \%$ as IPANN under predicted the stress (Fig. 4). On the contrary, the average reaction force at steady state from the prediction on strain stress behavior with generalized EM model is overestimated by about $8 \%$.

The same trend is found for the simulations with a deformation of $20 \%$ and $30 \%$ (Figs. 5-8). The equivalent plastic strain is distributed in a similar pattern for all three simulations (Figs. 6 and 8). The area of strains of different levels and the highest strain are related to the strain stress behavior. The reaction force also closely follows the previous observation. ${ }^{6)}$

All information presented from Figs. 3-8 can be summarized (Table 1) where the simulation is correlated to the strain-stress behavior observed from experiment and predicted from EM constitutive and IPANN models. It is found that the plastic strain and rolling reaction force at a specific strain are closely related to the product of the strain and stress, which is, therefore, an important parameter in the constitutive modeling and rollgap setup.

\subsection{Prediction of the Integrated FEM and IPANN Model}

The prediction of integrated FEM and ANN model will be compared with that of an IPANN model reported by

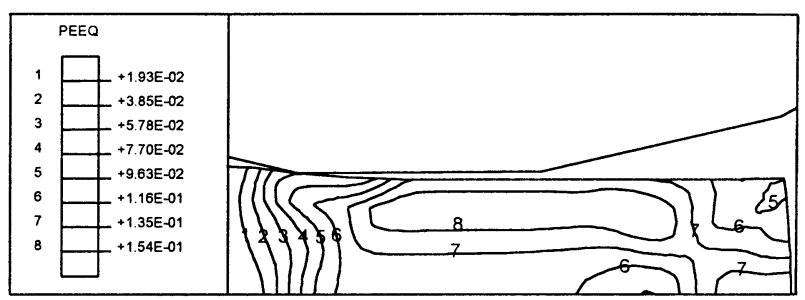

(a) Experiment

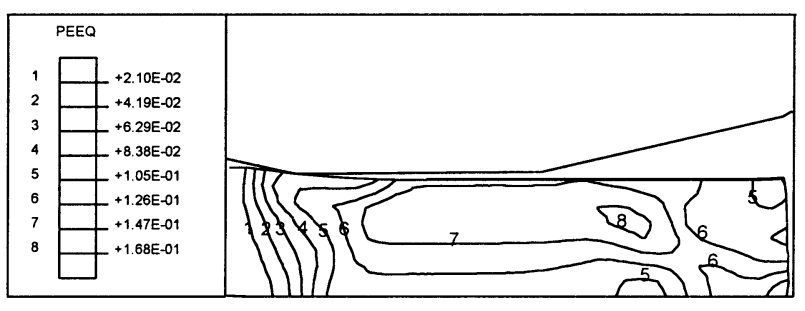

(b) Generalized EM model



(c) IPANN model

Fig. 3. Predicted equivalent plastic strain with a reduction of $10 \%$ in thickness.

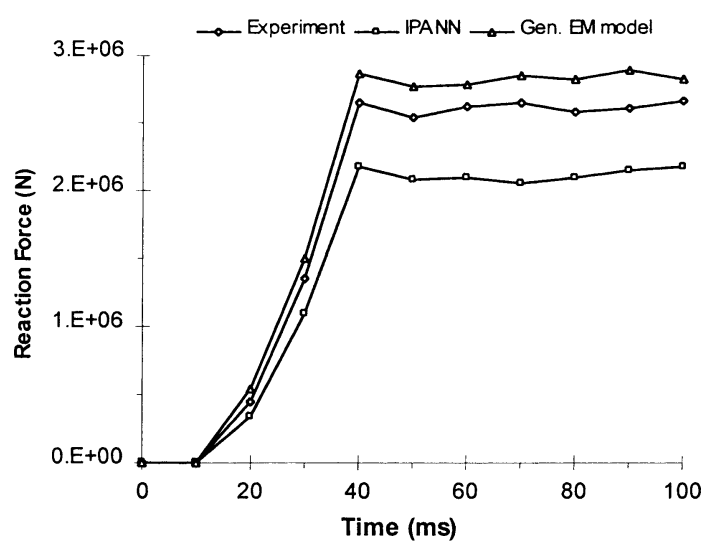

Fig 4. Comparison of the reaction force for simulations using strain-stress behaviour from experiment, EM and IPANN models $(10 \%)$.

Kong and Hodgson. ${ }^{3)}$ The same training techniques as used by Kong et al. ${ }^{5)}$ are used in this work. To show the improvement of the introduction of area in ANN model, the training and test data used in both predictions are the same and so are the training strategies and training stopping criteria.

For both the IPANN and integrated FEM and ANN models, steels with a carbon content of $0.0037,0.065,0.41$ and $0.79 \mathrm{wt} \% \mathrm{C}$ were used to train the model while steels with a carbon content of $0.017,0.21$ and $0.58 \mathrm{wt} \% \mathrm{C}$ were used to test the training where steel properties were listed in. ${ }^{5)}$ The 


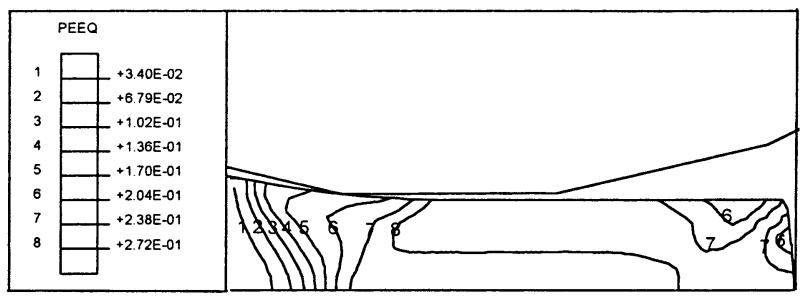

(a) Experiment

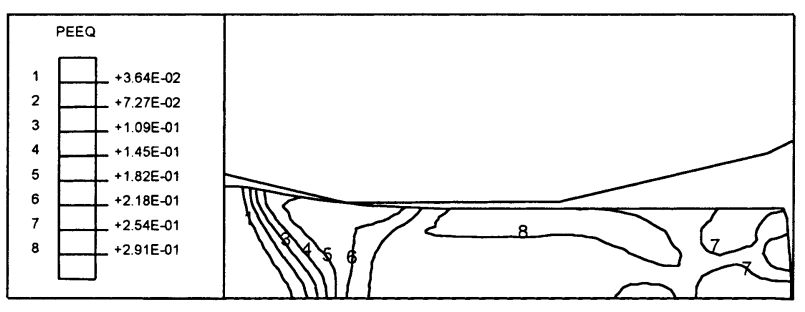

(b) Generalized EM model

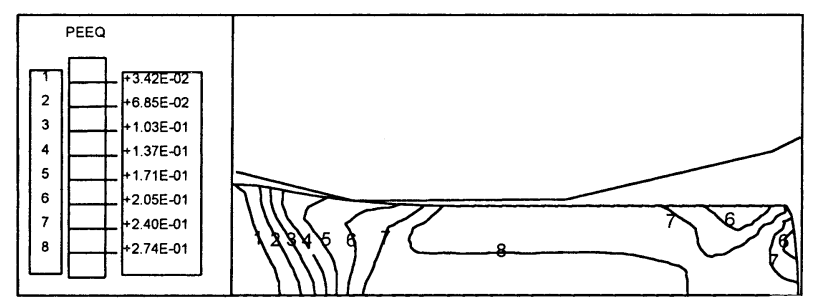

(c) IPANN model

Fig. 5. Predicted equivalent plastic strain with a reduction of $20 \%$ in thickness.

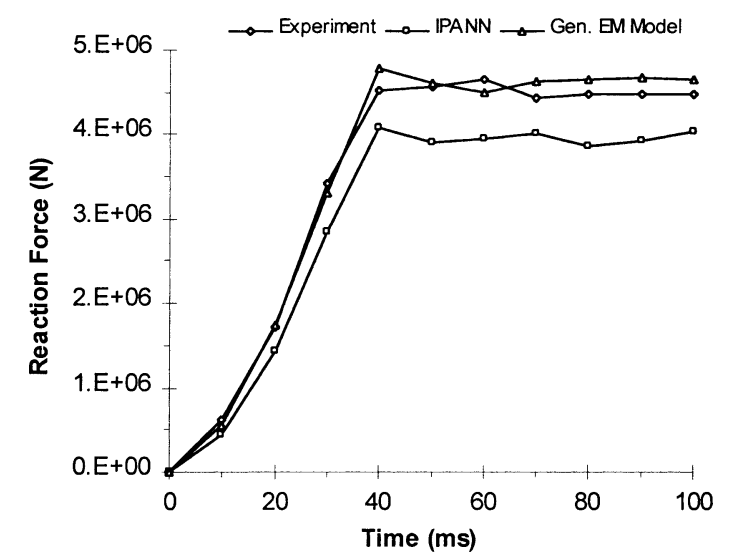

Fig. 6. Comparison of the reaction force for simulations using strain-stress behavior from experiment, EM and IPANN models $(20 \%)$.

prediction of IPANN model on the training data is shown in Fig. 9(a) with an average error of $1.7 \%$ and standard error of $6.3 \%$. The average and standard errors increase with an increase in the number of independent inputs and training data. The prediction on steels with a carbon content of $0.017,0.21$ and $0.58 \mathrm{wt} \% \mathrm{C}$ is shown in Fig. 9(b) with an average error of $4.4 \%$ and standard error of $8.8 \%$. Apart from test at low $Z$ (at low strain rates and high temperatures) where the stresses are low, the modified ANN model accurately predicts the stress-strain behavior in both the work hardening and dynamic recrystallisation regimes.

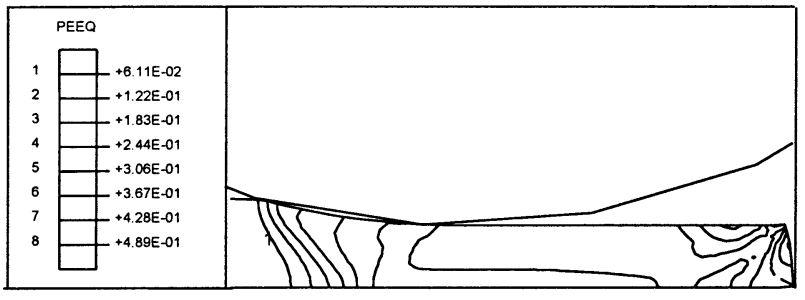

(a) Experiment

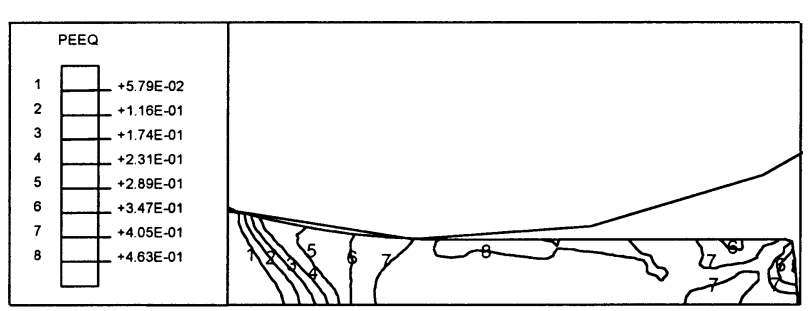

(b) Generalized EM model

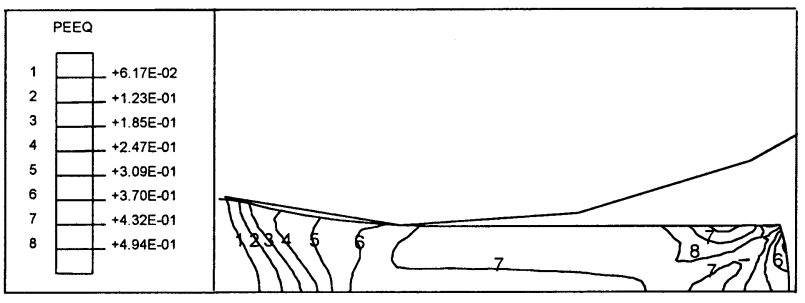

(c) IPANN model

Fig. 7. Predicted equivalent plastic strain with a reduction of $30 \%$ in thickness.

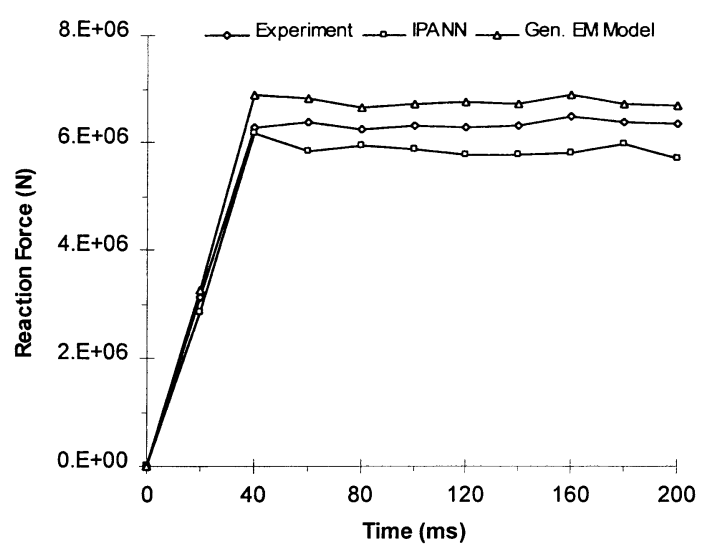

Fig. 8. Comparison of the reaction force for simulations using strain-stress behavior from experiment, EM and IPANN models (30\%).

Although the peak strain varies with carbon content in a complicate way, the IPANN model accurately predicts these characteristics.

The prediction of integrated FEM and ANN model is shown in Fig. 10. For training data set, the average error is $0.1 \%$ while the standard error is $7.1 \%$ (Fig. 10(a)). In comparison with the IPANN model, the standard error of the prediction of the integrated FEM and ANN model from training data set is higher. However, the model provides a more generalized prediction as the testing on steels with a carbon content of $0.017,0.21$ and $0.58 \mathrm{wt} \% \mathrm{C}$ is more accu- 
Table 1. Material properties and simulation results.

\begin{tabular}{|c|c|c|c|c|}
\hline \multicolumn{2}{|c|}{ Thickness reduction } & $10 \%$ & $20 \%$ & $30 \%$ \\
\hline $\operatorname{Area}\left(\sum_{0}^{\varepsilon_{0}} \sigma d \varepsilon\right)$ & $\begin{array}{l}\text { Exp. } \\
\text { Gen. EM } \\
\text { IPANN }\end{array}$ & $\begin{array}{l}6.2 \\
8.1 \\
4.8\end{array}$ & $\begin{array}{l}16.4 \\
18.4 \\
13.5\end{array}$ & $\begin{array}{l}28.6 \\
29.7 \\
24.0\end{array}$ \\
\hline Plastic Strain (Max.) & $\begin{array}{l}\text { Exp. } \\
\text { Gen. EM } \\
\text { IPANN }\end{array}$ & $\begin{array}{l}.16 \\
.16 \\
.17\end{array}$ & $\begin{array}{l}.28 \\
.30 \\
.28\end{array}$ & $\begin{array}{l}.51 \\
.48 \\
.51\end{array}$ \\
\hline Reaction Force N & $\begin{array}{l}\text { Exp. } \\
\text { Gen. EM } \\
\text { IPANN }\end{array}$ & $\begin{array}{l}2.6 \mathrm{e} 6 \\
2.8 \mathrm{e} 6 \\
2.1 \mathrm{e} 6\end{array}$ & $\begin{array}{l}4.5 \mathrm{e} 6 \\
4.6 \mathrm{e} 6 \\
4.0 \mathrm{e} 6\end{array}$ & $\begin{array}{l}6.3 \mathrm{e} 6 \\
6.8 \mathrm{e} 6 \\
5.9 \mathrm{e} 6\end{array}$ \\
\hline
\end{tabular}

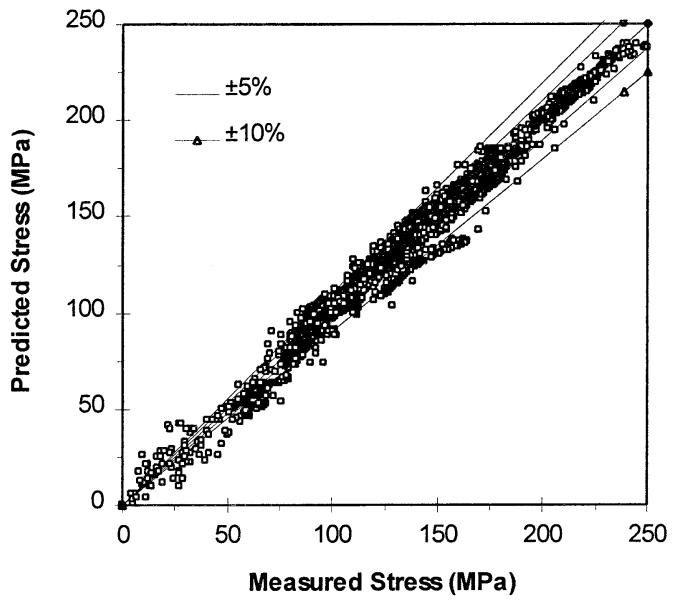

(a)

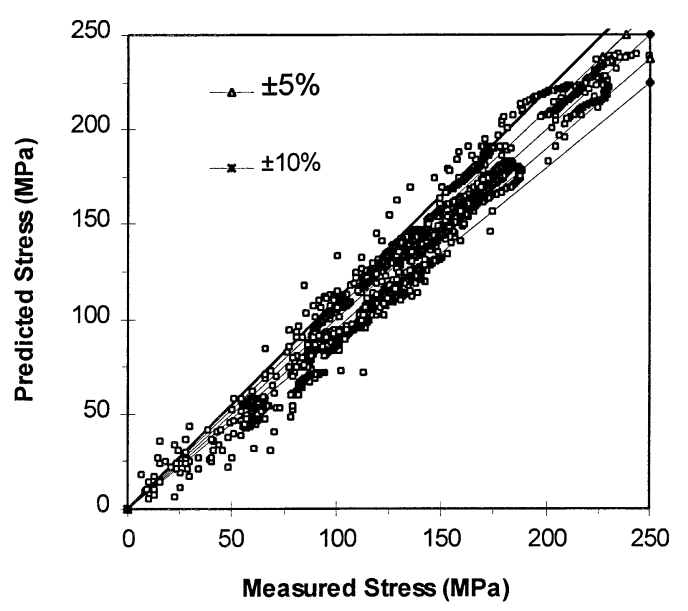

(b)

Fig. 9. Comparison of measured stress and that predicted using the IPANN model (a) training data (b) test data.

rate than the IPANN model with an average error of $2 \%$ and a standard error of $8.15 \%$ (Fig. 10(b)).



(a)

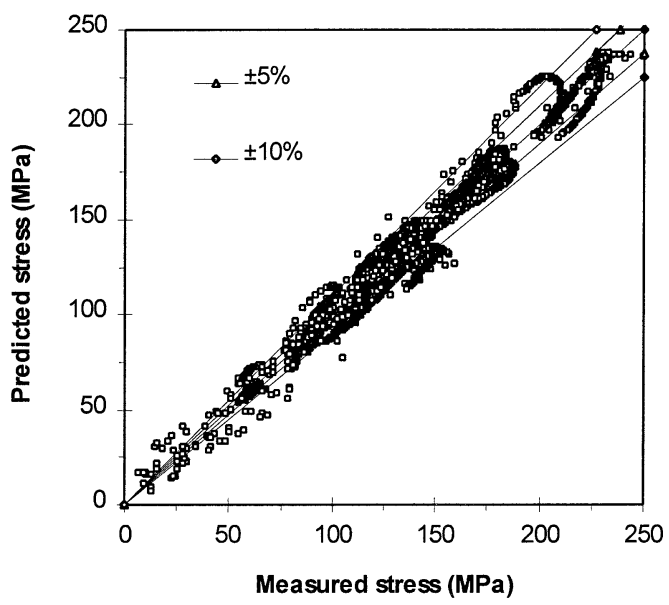

(b)

Fig. 10. Comparison of measured stress and that predicted using the integrated FEM and ANN model: (a) training data (b) test data.

\section{Conclusions}

The prediction accuracy of the EM constitutive and the IPANN models are evaluated using a commercial FEM code ABAQUS to provide the information for millgap setup. Using a 2-D simulation model, a plate of $40 \mathrm{~mm}$ in thickness is simulated with an average deformation of 10 , 20 and $30 \%$ respectively. It is found that the plastic strain and rolling reaction force at a specific strain are closely related to the product of the strain stress, which is therefore an important parameter in the constitutive modeling and rollgap setup. Therefore, the summary of the product of the strain and stress in the deformation range contributes most to the precise prediction of the models.

As the area of strain stress curve of steels is a very important factor influencing the rolling setup identified from the finite element simulation, it is introduced into the IPANN model as an additional input for training. It is found that the integrated FEM and ANN model provides a more generalized prediction than the IPANN model with the prediction on the test data set more accurate than other models. 
ISIJ International, Vol. 41 (2001), No. 7

\section{REFERENCES}

1) F. Kocks and H. Mecking: Acta Metall., 29 (1981), 1865.

2) P. Ludwik: Element der Tech. Mech., (1909), 32.

3) Y. L. Hwu, Y. T. Pan and J. G. Lenard: Steel Res., 67 (1996), 59.

4) D. C. Collinson, P. D. Hodgson and B. A. Parker: Modelling of Metal Rolling Processes, IOM, London, (1993), 283.

5) L. X. Kong, P. D. Hodgson and D. C. Collinson: ISIJ Int., 38 (1998),
1121.

6) L. X. Kong, P. D. Hodgson, K. K. Um and B. Wang: Modelling of Metal Rolling Processes 3, IOM, London, (1999), 116.

7) Y. Estrin and H. Mecking: Acta Metall., 32 (1984), 57.

8) M. Avrami: J. Chem. Phys, 7 (1939), 1103.

9) L. X. Kong and P. D. Hodgson: Advances Eng. Software, 31 (2000), 945. 